\title{
Immunohistochemical profile of odontogenic epithelium in developing dog teeth (Canis familiaris)
}

S. NEL ${ }^{a, \star}$, M. B. VAN HEERDEN ${ }^{a}$, G. STEENKAMP ${ }^{b}$ W. F. P. VAN HEERDEN ${ }^{a}$, S. C. $\mathrm{BOY}^{\mathrm{a}}$

${ }^{a}$ Department of Oral Pathology and Oral Biology, School of Dentistry, Faculty of Health Sciences, University of Pretoria, Oral and Dental Hospital, Bophelo Road, Pretoria, South Africa

${ }^{b}$ Department of Companion Animal Clinical Studies, Faculty of Veterinary Science, University of Pretoria, Old Soutpan Road, Onderstepoort, South Africa

Running Title: Immunohistochemical profile of odontogenic epithelium

"Corresponding author: Sulette Nel

PO Box 1266, Pretoria, 0001, South Africa

Tel.: +27 123192664

Fax: +27 123212225

E-mail address: sulette.nel@up.ac.za 


\section{ABSTRACT}

Tumours of the jaw bones and oral soft tissue are relatively common lesions in dogs. The aim of this study was to find cell markers to differentiate odontogenic epithelium from non-odontogenic epithelium for future research on the pathogenesis and pathology of odontogenic neoplasms in dogs. Keratin 14 and 19 staining was observed in odontogenic and non-odontogenic epithelium, while amelogenin and p75 neurotrophin receptor immunoreactivity was observed in certain odontogenic epithelial cells at various stages of development, but not in other epithelial cells. Calretinin staining was observed in the alveolar epithelial cells directly overlying the developing tooth germ in 28/39 sections $(71,8 \%)$, as well as the dental laminae in $30 / 35$ sections $(85,7 \%)$ and Serres rests in $24 / 28$ sections $(85,7 \%)$. Focal positivity was detected in the respiratory mucosa, some hair follicles and fusion epithelium of the palate but no calretinin staining was observed in other oral epithelial cells, and therefore calretinin has potential to be utilized as a marker to differentiate odontogenic form non-odontogenic epithelium.

Key words: amelogenin, calretinin, canine, dogs, keratin-14, keratin-19, odontogenesis, odontogenic epithelium, p75 neurotrophin receptor 
Dogs frequently present with oral epulides defined as tumours of the gingiva. Many of these can easily be identified as one of the commonly recognised odontogenic neoplasms. ${ }^{13,38,39}$ Problematic entities however do exist when the tumours do not have the classic histological features of any specific odontogenic tumour or cyst. In an attempt to elucidate the origin and character of these epulides of uncertain histogenesis, it is imperative to find molecular markers which could discriminate between odontogenic epithelium and other types of epithelium in the mouths of dogs. Such markers could then be utilized in future molecular research on the pathogenesis and pathology of odontogenic versus non-odontogenic neoplasms in dogs. To the best of our knowledge no in situ markers for odontogenic epithelium have been described in developing dog teeth. We therefore evaluated keratin 14 (K14) and 19 (K19), amelogenin, p75 neurotrophin receptor (p75NTR) and calretinin, which have been previously described as markers of odontogenic epithelium in developing human and rat odontogenic tissues, ${ }^{7-}$ 9,19,26 on developing dog teeth.

\section{Materials and methods}

Twenty four foetuses of large breed dogs were obtained under ethical clearance of the Animal Use and Care Committee of the Faculty of Veterinary Sciences, University of Pretoria, South Africa, from female dogs scheduled for elective pregnancy termination. The foetuses were fixed in $10 \%$ buffered formalin and the heads carefully cut into coronal sections, dehydrated and embedded in paraffin wax blocks. Those samples that contained calcified bone or dental hard tissues were decalcified in routine decalcifying solution (70ml $\mathrm{HNO}_{3}, 50 \mathrm{ml} \mathrm{HCL}, 880 \mathrm{ml}$ distilled water) for 60 minutes and then rinsed in running tap water for 60 minutes. Tissue specimens were sectioned at $3 \mu \mathrm{m}$, stained with haematoxylin and eosin (H\&E), and microscopically examined to select slides with 
well-formed enamel organs in which the respective odontogenic epithelial cells were morphologically clearly identifiable.

For the purpose of this study, the internal enamel epithelium (IEE) was defined as short columnar cells with centrally placed nuclei situated opposite undifferentiated ectomesenchymal cells of the dental papilla without any signs of odontoblast differentiation or dental hard tissue formation (Fig 1). Secretory ameloblasts were defined as tall columnar cells with prominent reversed polarity of the nuclei in close approximation to formed enamel and dentin (Fig. 1). Presecretory ameloblasts were defined as tall columnar cells with reversed polarity, but without the presence of dental hard tissue adjacent to these cells (Fig. 1). The cells of the outer enamel epithelium (OEE) were defined as low cuboidal cells with little cytoplasm at the outer periphery of the enamel organ (Fig. 1). The oral epithelium on the alveolar ridge area, from where the tooth germ originated, was referred to as the overlying alveolar epithelium (Fig. 1). The term dental lamina was used when there was continuity between the overlying oral epithelium and the developing tooth germ, while discrete islands of epithelial cells evident in this location were classified as Serres rests (Fig. 1).

The chosen tissue sections were dewaxed in xylene and hydrated with graded alcohol solutions. Heat induced epitope retrieval (HIER) in citric acid buffer, pH 6, was performed on the sections for the low affinity nerve growth factor receptor (NGFR) (gp75) (Novocastra NCL-NGFR, Novocastra Laboratories Ltd., Balliol Park West, Benton Lane, Newcastle Upon Tyne NE12 8EW United Kingdom), Cytokeratin 14 (Novocastra NCL-LL002) and Amelogenin (FL-191) (Santa Cruz Biotechnology sc-32892, Santa Cruz Biotechnology, Inc, 2145 Delaware Ave, Santa Cruz, California 95060) using a Pascal pressure chamber (Dako Cytomation, Inc. 6392 Via Real, Carpintera, California 93013 USA), according to the manufacturer's instructions. Sections were cooled for 20 minutes 
at room temperature and treated with hydrogen peroxide for 5 minutes at $3^{\circ} \stackrel{\circ}{ } \mathrm{C}$, to quench endogenous peroxidase activity. Sections stained for Calretinin (Novocastra NCL-Calretinin), and Cytokeratin 19 (Novocastra NCL-CK19) were first treated with hydrogen peroxide and then HIER was done in ethylenediaminetetraacetic acid (EDTA) buffer $\mathrm{pH} 8$ using the Pascal.

Sections were then incubated in their various anti serum: Cytokeratin 14, (1:60), Cytokeratin 19 (1:100), NGFR-gp75 (1:50), Calretinin (1:100) and Amelogenin (1:50) for 60 minutes at room temperature. All the sections, except the Amelogenin, were then incubated in Envision $^{\mathrm{TM}}+$ System-HRP Labelled Polymer Mouse (Dako K4000, Dako Cytomation Inc) for 35 minutes at room temperature. The Amelogenin sections were incubated in Envision ${ }^{\mathrm{TM}}+$ System-HRP Labelled Polymer Rabbit (Dako K4009) for 35 minutes at room temperature. All the sections were then stained with $\mathrm{AEC}+$ chromogen (Dako Cytomation Inc) for 4 minutes at $37^{\circ} \mathrm{C}$. Sections were counterstained with Haematoxylin, mounted with Faramount Aqueous Mounting Media (Dako S3025) and then examined microscopically.

\section{Results}

The exact time of gestation of the developing dog foetuses were unknown and the foetuses were classified in 2 groups based on the stage of odontogenic development. Foetuses 1-8 (group 1) only had tooth germs in the bud and cap stages of development with no ameloblast differentiation or dental hard tissue formation. The tooth germs of foetuses 9-24, (group 2), were in the bell stage of development with visible cell differentiation and hard tissue formation. In 15 cases, a rostral (anterior) and a caudal (posterior) section of the same foetus were used for comparison. In the remaining 9 cases, only one section complied with the inclusion criteria. A total of 39 sections were therefore harvested from the 24 foetuses. Although the number of tooth germs on a 
single section varied from 1 to 4 , only one, defined as the best representative of the bell stage in the given section, was chosen for analysis.

\section{Calretinin}

Group 1 foetuses showed calretinin staining of the OEE but no calretinin staining was observed in the OEE of group 2, IEE, ameloblasts, odontoblasts or epithelial cells of the stellate reticulum or stratum intermedium at any stage of development. Thirty of the 35 dental laminae evaluated stained positive for calretinin (85.7\%). Twenty four of 28 cases with Serres rests showed positive staining in the epithelial rests (85.7\%). The overlying alveolar epithelium, in close approximation to the lamina and Serres rests, stained positive in 28 of the 39 cases (71.8\%) evaluated (Fig. 2).

\section{Keratin 14}

Intense and diffuse K14 staining was present in the IEE of $5 / 5$ of group 1 foetuses while the group 2 foetuses mainly showed focal staining in the IEE in 20/22 (91\%) of cases examined. No staining of pre-secretory ameloblasts could be seen in group 2 while intense and diffuse staining of the secretory ameloblasts was seen in all cases. The staining intensity of the secretory ameloblasts decreased as enamel secretion continued. The OEE in all sections (36/36) stained positive, although the distribution and intensity varied. Intense and diffuse staining of the stellate reticulum (6/6) and stratum intermedium epithelium (5/5) was seen in group 1. In group 2, 15/30 (50\%) of cases showed positive staining in the stellate reticulum and 14/29 (48\%) showed positive staining in the stratum intermedium epithelium. Intense staining patterns were observed throughout the full thickness of the alveolar epithelium, dental laminae and Serres rests in all cases examined. No positive staining was detected in any of the odontoblasts present in the sections (Fig. 3). 


\section{Keratin 19}

In both group 1 and 2 the epithelial cells of the enamel organs of all the sections stained positive with variable intensities and distribution patterns. The IEE cells of group 1 exhibited a heterogeneous pattern of staining as staining intensities varied. In group 2, diffuse expression of K19 was observed in the IEE cells, except in the cervical loop region. The pre-secretory and secretory ameloblasts stained diffusely positive in all cases although with varied intensities. Positive staining was observed in the full thickness of the overlying alveolar epithelial cells in all of the group 1 foetuses, but in group 2 it was mainly restricted to the superficial epithelial cell layers. Diffuse staining was observed in all the dental laminae and Serres rests in all cases examined. No positive staining was detected in any of the odontoblasts present on the sections (Fig. 4).

\section{Amelogenin}

No amelogenin staining was present in group 1. In group 2, small amounts of amelogenin were observed in presecretory ameloblasts seen in close approximation to the cell nucleus. Diffuse granular staining for the amelogenin protein was observed in all secretory ameloblasts present in group 2 sections. No staining was detected in the IEE, OEE, stellate reticulum, stratum intermedium, dental laminae, Serres rests or alveolar epithelium of both groups. Of the 30 evaluated group 2 sections, 6 revealed weak staining in some odontoblasts $(20 \%)$. Staining seemed to be localized to the intercellular spaces between the odontoblasts and other pulp cells. Staining of these sections was repeated but the same results were obtained. No amelogenin staining was observed in or around odontoblasts at advanced stages of odontogenesis (Fig. 5).

\section{p75NTR}

No epithelial staining was observed in bud stage dental organs. Focal positivity was observed in the IEE of 5/5 cap stage germs as well as in the region of the cervical loops 
of 9/28 (32\%) bell stage tooth organs. No staining was observed in OEE, stellate reticulum, stratum intermedium, ameloblasts, dental lamina, Serres rests or overlying alveolar epithelium. In 22/27 (81\%) cases, some pre-odontoblasts stained positive. As soon as the odontoblasts differentiated, the positivity disappeared. Faint positivity was observed throughout the pulp cells in $3 / 5(60 \%)$ cap stage tooth germs but during the bell stage in $21 / 28(75 \%)$ of tooth germs staining was restricted to the apical region of the pulp only. The dental follicle cells stained diffusely positive throughout the evaluated sections.

\section{Discussion}

Calretinin staining could only be demonstrated in the epithelium of the dental laminae, Serres rests and associated overlying alveolar epithelium (Fig. 2, Table 1), while Mistry and co-workers described different levels of calretinin expression in the dental lamina, OEE, stellate reticulum, stratum intermedium, IEE and ameloblasts of rat teeth. ${ }^{22}$ To the best of our knowledge, their study was the only one describing calretinin expression in developing odontogenic tissue. They suggested calretinin, a calcium binding protein, to play a role in enamel formation as calretinin expression remained negative in the molar teeth over the cusp tips, where enamel is never formed. ${ }^{22}$ Differences in antibodies and techniques used, species differences, ${ }^{2,32}$ and differences in the developmental stages of the foetuses examined could offer some possible explanations for the differences in results found ${ }^{34}$ Focal positivity was detected in the respiratory mucosa, some hair follicles and fusion epithelium of the palate in the current study. We hypothesize that calretinin could be a useful marker for odontogenic epithelial cells as the positive staining found in the overlying alveolar epithelium could not be detected in the rest of the oral epithelial cells. From this study we propose that calretinin should be investigated further in this respect. 
$\mathrm{K} 14$ is a type I intermediate filament (IF) said to confer physical resilience to basal epithelial cells. ${ }^{6}$ It is the main IF of odontogenic epithelium and a marker often utilized in the detection of ameloblasts and IEE. Immunohistochemical studies have shown strong K14 positivity in IEE of human foetuses at the early bell stage ${ }^{9}$ with weaker staining in fully differentiated ameloblasts at a later developmental stage..$^{9,44}$ In contrast, others have shown the IEE cells of rat tooth germs in the earlier stages to have weak expression of $\mathrm{K} 14$ with more intense staining in pre-ameloblasts and even more so in fully differentiated ameloblasts. ${ }^{35}$ The staining patterns of K14 in the current study are unique. Intense and diffuse staining was observed in the IEE of all the group 1 foetuses while the same cells in the group 2 foetuses revealed only focal staining in 20/22 (91\%) cases. No K14 staining was seen in any presecretory ameloblasts but this changed to intense and diffuse staining of all secretory ameloblasts in all cases (Fig. 3), which again decreased as enamel secretion continued. This phenomenon of decreased and ultimately no K14 staining in the presecretory ameloblasts was difficult to explain. K14 has been described as an anchorage protein that might have a role in supporting and preserving the epithelial-mesenchymal interactions. ${ }^{9,11}$ It has been speculated that ameloblasts lose $\mathrm{K} 14$ to disengage its anchorage in order to migrate for enamel matrix deposition. ${ }^{9}$ The dynamic nature of intermediary filament expression as well as the occurrence of selective or complete masking of the IF determinant ${ }^{12}$ could offer more explanations for our observed staining pattern. However, the exact mechanism of the in vivo regulation of keratins, still remain to be determined. It is possible that the various author's definitions of pre-ameloblasts, presecretory ameloblasts, and secretory ameloblasts differ from one another and therefore offers another reason for the differences in the respective results found. This study showed K14 expression throughout the odontogenic epithelium with no staining in the dental mesenchyme (Table 
1), but staining of the odontogenic epithelium was not exclusive as positivity was also observed in other types of epithelium present on the coronal sections (oral epithelium, skin, salivary glands and ducts, and some respiratory mucosa).

K19, another type I IF, has been described in the epithelium of the stellate reticulum and stratum intermedium, secretory ameloblasts as well as ameloblasts in the maturation stage of monkeys (Macaca mulatta and Macaca nemestrina). ${ }^{21}$ In human foetuses, K19 (in association with K8) has been described in all cells of the dental lamina and enamel organ ${ }^{9,16}$ with intense positive staining in the fully differentiated ameloblasts. ${ }^{9}$ For this reason, $\mathrm{K} 19$ has been postulated to be a marker of ameloblast differentiation. ${ }^{9}$ Our study agreed with others which found K19 staining patterns in the IEE to vary from weak single cell to intense and diffuse but with constant diffuse and intense staining of the presecretory and secretory ameloblasts (Fig. 4). ${ }^{9}$ Positive staining for K19 was observed in hair follicles, salivary gland ducts, respiratory mucosa and oral epithelium and therefore, K19 did not stain odontogenic epithelium exclusively and could not be regarded as a specific marker for odontogenic epithelium (Table 1). Because the pattern of staining for both K14 and K19 appeared to change with stage of development, it is possible that yet another profile would be seen in adult tissues and should be investigated further.

Amelogenins are enamel matrix proteins secreted by ameloblasts and constitute over $90 \%$ of the developing enamel extracellular matrix during odontogenesis. ${ }^{10,25,36}$ In the past, amelogenin was widely accepted as an enamel-specific protein ${ }^{1,17,30,37,42}$ which was proposed and used as a stage specific marker for ameloblast differentiation. Its expression was not detected in IEE while expression was found in pre-ameloblasts and ameloblasts. ${ }^{8,35,44}$ However, amelogenin was detected in hamster mantle dentin, ${ }^{18}$ which was proposed to be the result of diffusion or translocation of amelogenin into the predentin and pre-odontoblast layer with endocytosis of the amelogenin protein by the 
odontoblasts. ${ }^{18,27}$ It has also been suggested that the presence of trace amounts of amelogenin splice products in dentin could be the result of contamination. ${ }^{28}$ Since then, amelogenin mRNA was detected in odontoblasts of porcine and rat teeth ${ }^{29,31}$ and in 2006 it was suggested that odontoblasts actually synthesize and secrete amelogenins after in situ hybridization and immunohistochemistry studies on human odontogenic tissue. ${ }^{45}$ The mRNA of splice forms of amelogenin have been detected in periodontal ligament cells (cementoblasts) of wild-type mice ${ }^{14}$ and recently amelogenin mRNA and protein was described in dog and rat osteoblasts, osteoclasts, in some osteocytes and also in articular cartilage chondrocytes. ${ }^{15}$ It was therefore proposed that amelogenin is also expressed in mesenchymal cells and is not only an ameloblast-specific protein as previously believed. ${ }^{14,15}$

Expression of the amelogenin protein in this study was similar to previous studies which found it to vary from only small amounts in presecretory ameloblasts, ${ }^{17,30}$ to diffuse positivity throughout differentiated ameloblasts in the secretory stage (Fig. 5). ${ }^{17,30,37}$ Single cell positivity of odontoblasts was observed in $6 / 30(20 \%)$ sections with odontoblasts present. On close examination, the staining appeared to be localized to the intercellular spaces. It is therefore possible that the single cell positivity observed in the odontoblasts in our study could in fact be artifactual. The amelogenin protein actually located outside the cell could have been misinterpreted as intracellular staining due to superimposition on the slide. No staining was observed in odontoblasts at advanced stages of odontogenesis and the single cells that did seem to stain were associated with the mantle dentin only. Although staining has been described in other tissues before, ${ }^{15}$ amelogenin was only detected in odontogenic tissues in this study and seems to be a promising marker to distinguish between odontogenic and nonodontogenic tissues in dogs (Table 1). 
In 1985 it was observed that two classes of NGFRs exists, originally described as high and low affinity receptors. ${ }^{4}$ Today it is better known as the tropomyosin-related kinase (Trk) tyrosine kinase receptors (high affinity receptor) and the p75NTR (low affinity receptor). ${ }^{33}$ p75NTR has been described in epithelial cells of the growing tooth germ as well as the associated ectomesenchymal cells of rat teeth. ${ }^{5,23,24}$ In the bell stage, staining has been observed in proliferating cells of the IEE,,$^{5,23,43}$ some cells of the stratum intermedium and in pre-odontoblasts/polarizing odontoblasts., ${ }^{5,23,24}$ More recent studies revealed immunoreactivity against p75NTR to be restricted to the IEE, the dental papilla and the dental follicle of rat incisors and therefore p75NTR has been used as a marker for IEE cells. ${ }^{19,26}$ In the current study p75NTR expression was restricted to focal regions of the IEE in 14/33 sections (42\%) and no staining was observed in other epithelial cells (Table 1). It could therefore be utilized as a marker to differentiate between odontogenic epithelium in the early stages of development versus nonodontogenic epithelium. The widespread staining observed in the mesenchymal tissue does not permit p75NTR to be a specific marker for odontogenic epithelium although the morphological differences between epithelium and mesenchymal tissue would aid in the histological investigation. The precise function of p75NTR expression in the IEE cells remains a speculative topic.

Odontogenic tumours may originate from the epithelial or ectomesenchymal cells of the developing tooth germ or its remnants. ${ }^{20}$ Most of the odontogenic tumours are found in adult dogs where all that is left of the odontogenic epithelium is the Serres and Malassez odontogenic epithelium rests and in certain cases the reduced enamel epithelium associated with an unerupted (or impacted) tooth. One can therefore assume that the odontogenic tumours in adult dogs would most probably originate from these epithelial remnants in the gingiva and the periodontal ligament space. ${ }^{3}$ As it was shown in this 
study, the expression profile of certain markers was altered in group 2 compared to group 1 foetuses in many respects. This expression profile may change even more in post-natal and adult tissue as previously indicated..$^{34,40,41,43}$ Therefore, it would be possible that a marker could retain its expression in Serres- and Malassez rests, but could loose its expression in oral epithelial cells that lost their odontogenic potential. We propose that the oral epithelial cells would gain more mature differentiated characteristics in adult tissue as opposed to the Serres- and Malassez rests that may retain their original foetal characteristics and expression profiles, as they are quiescent remnants of foetal developmental cells. Finding a suitable marker that will stain the odontogenic rests without staining the remainder of the oral epithelial cells in adult tissue could aid in finding a marker to distinguish between tumours originating from odontogenic tissue compared to those taking origin from the adjacent oral epithelium. The expression of the same markers that were used in this study should therefore be tested in adult dog tissue, specifically to evaluate the immunoreactivity of the Serres and Malassez odontogenic epithelium rests and associated gingiva.

\section{Acknowledgements}

We would like to thank the Veterinary Academic Hospital at Onderstepoort and Dr. MF Visser (Lakeside Veterinary Clinic) for providing us with the canine foetuses used in this study. 


\section{References}

1 Abiko Y, Murata M, Ito Y, Taira T, Nishimura M, Arisue M, Inoue T, Shimono M, Kuboki Y, Kaku T: Immunohistochemical localization of amelogenin in human odontogenic tumors, using a polyclonal antibody against bovine amelogenin. Med Electron Microsc 34: 185-189, 2001

2 Andressen C, Blumcke I, Celio MR: Calcium-binding proteins: selective markers of nerve cells. Cell Tissue Res 271: 181-208, 1993

3 Buchner A, Sciubba JJ: Peripheral epithelial odontogenic tumors: a review. Oral Surg Oral Med Oral Pathol 63: 688-697, 1987

4 Buxser S, Puma P, Johnson GL: Properties of the nerve growth factor receptor. Relationship between receptor structure and affinity. J Biol Chem 260: 19171926, 1985

5 Byers MR, Schatteman GC, Bothwell M: Multiple functions for NGF receptor in developing, aging and injured rat teeth are suggested by epithelial, mesenchymal and neural immunoreactivity. Development 109: 461-471, 1990

6 Chan Y, Anton-Lamprecht I, Yu QC, Jackel A, Zabel B, Ernst JP, Fuchs E: A human keratin 14 "knockout": the absence of K14 leads to severe epidermolysis bullosa simplex and a function for an intermediate filament protein. Genes Dev 8: 25742587, 1994

7 Crivelini MM, de Araujo VC, de Sousa SO, de Araujo NS: Cytokeratins in epithelia of odontogenic neoplasms. Oral Dis 9: 1-6, 2003

8 DenBesten PK, Machule D, Zhang Y, Yan Q, Li W: Characterization of human primary enamel organ epithelial cells in vitro. Arch Oral Biol 50: 689-694, 2005

9 Domingues MG, Jaeger MM, Araujo VC, Araujo NS: Expression of cytokeratins in human enamel organ. Eur J Oral Sci 108: 43-47, 2000 
10 Fincham AG, Moradian-Oldak J, Simmer JP: The structural biology of the developing dental enamel matrix. J Struct Biol 126: 270-299, 1999

11 Fuchs E, Cleveland DW: A structural scaffolding of intermediate filaments in health and disease. Science 279: 514-519, 1998

12 Fuchs E, Weber K: Intermediate filaments: structure, dynamics, function, and disease. Annu Rev Biochem 63: 345-382, 1994

13 Gardner DG: Epulides in the dog: a review. J Oral Pathol Med 25: 32-37, 1996

14 Hatakeyama J, Sreenath T, Hatakeyama Y, Thyagarajan T, Shum L, Gibson CW, Wright JT, Kulkarni AB: The receptor activator of nuclear factor-kappa B ligandmediated osteoclastogenic pathway is elevated in amelogenin-null mice. J Biol Chem 278: 35743-35748, 2003

15 Haze A, Taylor AL, Blumenfeld A, Rosenfeld E, Leiser Y, Dafni L, Shay B, Gruenbaum-Cohen Y, Fermon E, Haegewald S, Bernimoulin JP, Deutsch D: Amelogenin expression in long bone and cartilage cells and in bone marrow progenitor cells. Anat Rec (Hoboken) 290: 455-460, 2007

16 Heikinheimo K, Hormia M, Stenman G, Virtanen I, Happonen RP: Patterns of expression of intermediate filaments in ameloblastoma and human fetal tooth germ. J Oral Pathol Med 18: 264-273, 1989

17 Hu JC, Sun X, Zhang C, Simmer JP: A comparison of enamelin and amelogenin expression in developing mouse molars. Eur J Oral Sci 109: 125-132, 2001

18 Karg HA, Burger EH, Lyaruu DM, Woltgens JH, Bronckers AL: Gene expression and immunolocalisation of amelogenins in developing embryonic and neonatal hamster teeth. Cell Tissue Res 288: 545-555, 1997

19 Kawano S, Saito M, Handa K, Morotomi T, Toyono T, Seta Y, Nakamura N, Uchida T, Toyoshima K, Ohishi M, Harada H: Characterization of dental epithelial 
progenitor cells derived from cervical-loop epithelium in a rat lower incisor. J Dent Res 83: 129-133, 2004

20 Lu Y, Xuan M, Takata T, Wang C, He Z, Zhou Z, Mock D, Nikai H: Odontogenic tumors. A demographic study of 759 cases in a Chinese population. Oral Surg Oral Med Oral Pathol Oral Radiol Endod 86: 707-714, 1998

21 Massoth DL, Dale BA: Immunohistochemical study of structural proteins in developing junctional epithelium. J Periodontol 57: 756-763, 1986

22 Mistry D, Altini M, Coleman HG, Ali H, Maiorano E: The spatial and temporal expression of calretinin in developing rat molars (Rattus norvegicus). Arch Oral Biol 46: 973-981, 2001

23 Mitsiadis TA, Couble P, Dicou E, Rudkin BB, Magloire H: Patterns of nerve growth factor (NGF), proNGF, and p75 NGF receptor expression in the rat incisor: comparison with expression in the molar. Differentiation 54: 161-175, 1993

24 Mitsiadis TA, Luukko K: Neurotrophins in odontogenesis. Int J Dev Biol 39: 195-202, 1995

25 Moradian-Oldak J: Amelogenins: assembly, processing and control of crystal morphology. Matrix Biol 20: 293-305, 2001

26 Morotomi T, Kawano S, Toyono T, Kitamura C, Terashita M, Uchida T, Toyoshima K, Harada $\mathrm{H}$ : In vitro differentiation of dental epithelial progenitor cells through epithelial-mesenchymal interactions. Arch Oral Biol 50: 695-705, 2005

27 Nakamura M, Bringas P, Jr., Nanci A, Zeichner-David M, Ashdown B, Slavkin HC: Translocation of enamel proteins from inner enamel epithelia to odontoblasts during mouse tooth development. Anat Rec 238: 383-396, 1994

28 Nebgen DR, Inoue H, Sabsay B, Wei K, Ho CS, Veis A: Identification of the chondrogenic-inducing activity from bovine dentin (bCIA) as a low-molecularmass amelogenin polypeptide. J Dent Res 78: 1484-1494, 1999 
29 Oida S, Nagano T, Yamakoshi Y, Ando H, Yamada M, Fukae M: Amelogenin gene expression in porcine odontoblasts. J Dent Res 81: 103-108, 2002

30 Papagerakis P, Hotton D, Lezot F, Brookes S, Bonass W, Robinson C, Forest N, Berdal A: Evidence for regulation of amelogenin gene expression by 1,25dihydroxyvitamin D(3) in vivo. J Cell Biochem 76: 194-205, 1999

31 Papagerakis P, MacDougall M, Hotton D, Bailleul-Forestier I, Oboeuf M, Berdal A: Expression of amelogenin in odontoblasts. Bone 32: 228-240, 2003

32 Redecker P, Cetin Y, Korf HW: Differential immunocytochemical localization of calretinin in the pineal gland of three mammalian species. J Neurocytol 25: 9-18, 1996

33 Roux PP, Barker PA: Neurotrophin signaling through the p75 neurotrophin receptor. Prog Neurobiol 67: 203-233, 2002

34 Schwaller B, Celio MR, Hunziker W: Alternative splicing of calretinin mRNA leads to different forms of calretinin. Eur J Biochem 230: 424-430, 1995

35 Tabata MJ, Matsumura T, Liu JG, Wakisaka S, Kurisu K: Expression of cytokeratin 14 in ameloblast-lineage cells of the developing tooth of rat, both in vivo and in vitro. Arch Oral Biol 41: 1019-1027, 1996

36 Termine JD, Belcourt AB, Christner PJ, Conn KM, Nylen MU: Properties of dissociatively extracted fetal tooth matrix proteins. I. Principal molecular species in developing bovine enamel. J Biol Chem 255: 9760-9768, 1980

37 Torres-Quintana MA, Gaete M, Hernandez M, Farias M, Lobos N: Ameloblastin and amelogenin expression in posnatal developing mouse molars. J Oral Sci 47: 2734,2005

38 Ueki H, Sumi A, Takaishi H, Ito H, Oyamada T, Yoshikawa H: Malignant ameloblastic fibro-odontoma in a dog. Vet Pathol 41: 183-185, 2004 
39 Watanabe K, Kadosawa T, Ishiguro T, Takagi S, Ochiai K, Kimura T, Okumura M, Fujinaga T: Odontogenic cysts in three dogs: one odontogenic keratocyst and two dentigerous cysts. J Vet Med Sci 66: 1167-1170, 2004

40 Wheeler EF, Bothwell M: Spatiotemporal patterns of expression of NGF and the lowaffinity NGF receptor in rat embryos suggest functional roles in tissue morphogenesis and myogenesis. J Neurosci 12: 930-945, 1992

41 Wheeler EF, Gong H, Grimes R, Benoit D, Vazquez L: p75NTR and Trk receptors are expressed in reciprocal patterns in a wide variety of non-neural tissues during rat embryonic development, indicating independent receptor functions. J Comp Neurol 391: 407-428, 1998

42 Wurtz T, Lundmark C, Christersson C, Bawden JW, Slaby I, Hammarstrom L: Expression of amelogenin mRNA sequences during development of rat molars. J Bone Miner Res 11: 125-131, 1996

43 Yan Q, Johnson EM, Jr.: An immunohistochemical study of the nerve growth factor receptor in developing rats. J Neurosci 8: 3481-3498, 1988

44 Yan Q, Zhang Y, Li W, DenBesten PK: Differentiation of human ameloblast-lineage cells in vitro. Eur J Oral Sci 114 Suppl 1: 154-158; discussion 164-155, 380-151, 2006

45 Ye L, Le TQ, Zhu L, Butcher K, Schneider RA, Li W, Besten PK: Amelogenins in human developing and mature dental pulp. J Dent Res 85: 814-818, 2006 


\section{Figure legends}

Fig. 1. Diagrammatic representation of cells present in developing tooth germ. $a=I E E$, $\mathrm{b}=$ presecretory ameloblasts, $\mathrm{c}=$ secretory ameloblasts, $\mathrm{d}=\mathrm{OEE}, \mathrm{e}=$ overlying alveolar epithelium, $\mathrm{f}=$ Serres rests and $\mathrm{g}=$ dental lamina.

Fig. 2. Positive calretinin staining of a dental lamina (bold arrow) and basal cells of the overlying alveolar epithelium (thin arrow).

Fig. 3. Strong positive keratin 14 staining in the overlying alveolar epithelium, oral epithelium, dental lamina and secretory ameloblasts.

Fig. 4. Positive keratin 19 staining in the overlying alveolar epithelium, oral epithelium, dental lamina and all the epithelial cells of the enamel organ.

Fig. 5. Positive amelogenin staining in secretory ameloblasts (bold arrow) and enamel (thin arrow). 
Table 1. Summary of the expression patterns observed in dog odontogenic and non-odontogenic tissue

\begin{tabular}{|c|c|c|c|c|c|}
\hline & Calretinin & Keratin 14 & Keratin 19 & Amelogenin & p75 NTR \\
\hline IEE & - & ++ & ++ & - & + \\
\hline Ameloblasts & - & $\begin{array}{c}++ \\
\text { Secretory }\end{array}$ & $\begin{array}{c}++ \\
\text { Presecretory } \\
\text { and } \\
\text { Secretory }\end{array}$ & $\begin{array}{c}++ \\
\text { Presecretory } \\
\text { and } \\
\text { Secretory }\end{array}$ & - \\
\hline $\begin{array}{l}\text { Alveolar } \\
\text { epithelium/ } \\
\text { Dental } \\
\text { lamina/ } \\
\text { Serres Rests }\end{array}$ & ++ & ++ & ++ & - & - \\
\hline Odontoblasts & - & - & - & $\begin{array}{c}+ \\
\text { During } \\
\text { Mantle } \\
\text { Dentin } \\
\text { formation }\end{array}$ & $\begin{array}{c}+ \\
\text { Pre- } \\
\text { odontoblasts }\end{array}$ \\
\hline $\begin{array}{l}\text { Non- } \\
\text { odontogenic } \\
\text { epithlium }\end{array}$ & - & ++ & ++ & - & - \\
\hline
\end{tabular}

$+\quad$ Focal positive staining observed

$+\quad$ Diffuse positive staining observed 
No positive staining observed 

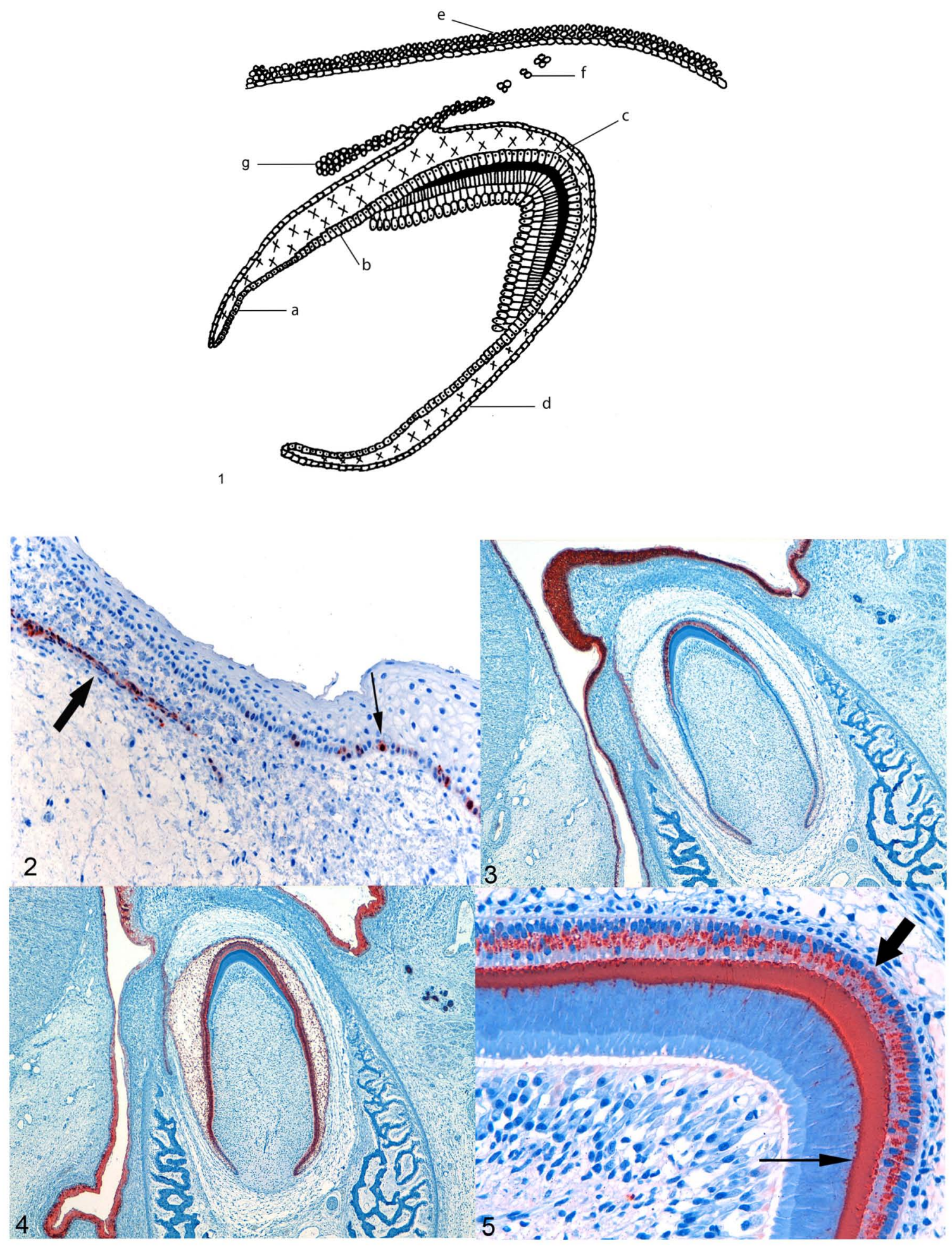